\title{
White matter hyperintensities classified according to intensity and spatial location reveal specific associations with cognitive performance
}

\author{
Luca Melazzini ${ }^{\text {a,b, * }}$, Clare E. Mackay b,c,d, Valentina Bordin ${ }^{\text {b,e }}$, Sana Suri ${ }^{\text {c,f }}$, Eniko Zsoldos b,c,f, \\ Nicola Filippini $^{\text {c }}$, Abda Mahmood ${ }^{\mathrm{f}}$, Vaanathi Sundaresan ${ }^{\mathrm{b}}$, Marina Codari ${ }^{\mathrm{g}}$, Eugene Duff ${ }^{\mathrm{b}, \mathrm{h}}$, \\ Archana Singh-Manoux ${ }^{i, j}$, Mika Kivimäki ${ }^{j}$, Klaus P. Ebmeier ${ }^{f}$, Mark Jenkinson ${ }^{b}$, \\ Francesco Sardanelli ${ }^{\mathrm{a}, \mathrm{k}}$, Ludovica Griffanti ${ }^{\mathrm{b}, \mathrm{c}}$ \\ a Department of Biomedical Sciences for Health, Università degli Studi di Milano, Milan, Italy \\ ${ }^{\mathrm{b}}$ Wellcome Centre for Integrative Neuroimaging, Oxford Centre for Functional MRI of the Brain, Nuffield Department of Clinical Neurosciences, University of Oxford, \\ Oxford, UK \\ ${ }^{c}$ Wellcome Centre for Integrative Neuroimaging, Oxford Centre for Human Brain Activity, Department of Psychiatry, University of Oxford, Oxford, UK \\ d Oxford Health NHS Foundation Trust, Oxford, UK \\ ${ }^{\mathrm{e}}$ Department of Electronics, Information and Bioengineering, Politecnico di Milano, Milan, Italy \\ ${ }^{\mathrm{f}}$ Department of Psychiatry, Warneford Hospital, University of Oxford, Oxford, UK \\ ${ }^{g}$ Department of Radiology, Stanford University School of Medicine, Stanford, USA \\ ${ }^{\mathrm{h}}$ Department of Paediatrics, University of Oxford, Oxford, UK \\ ${ }^{\mathrm{i}}$ INSERM U1153, Epidemiology of Ageing and Neurodegenerative Diseases, Université de Paris, Paris, France \\ ${ }^{\mathrm{j}}$ Department of Epidemiology and Public Health, University College London, London, UK \\ ${ }^{\mathrm{k}}$ Department of Radiology, IRCCS Policlinico San Donato, Milan, Italy
}

\section{A R T I C L E I N F O}

\section{Keywords:}

Aging

Cerebral small vessel diseases

Cognition

Magnetic resonance imaging

White matter

\begin{abstract}
A B S T R A C T
White matter hyperintensities (WMHs) on $\mathrm{T}_{2}$-weighted images are radiological signs of cerebral small vessel disease. As their total volume is variably associated with cognition, a new approach that integrates multiple radiological criteria is warranted. Location may matter, as periventricular WMHs have been shown to be associated with cognitive impairments. WMHs that appear as hypointense in $\mathrm{T}_{1}$-weighted images $\left(\mathrm{T}_{1} \mathrm{w}\right)$ may also indicate the most severe component of WMHs. We developed an automatic method that sub-classifies WMHs into four categories (periventricular/deep and $\mathrm{T}_{1} \mathrm{w}$-hypointense/non $\mathrm{T}_{1} \mathrm{w}$-hypointense) using MRI data from 684 community-dwelling older adults from the Whitehall II study. To test if location and intensity information can impact cognition, we derived two general linear models using either overall or subdivided volumes. Results showed that periventricular $\mathrm{T}_{1} \mathrm{w}$-hypointense WMHs were significantly associated with poorer performance in the trail making A ( $p=0.011)$, digit symbol $(\mathrm{p}=0.028)$ and digit coding $(\mathrm{p}=0.009)$ tests. We found no association between total WMH volume and cognition. These findings suggest that sub-classifying WMHs according to both location and intensity in $\mathrm{T}_{1} \mathrm{w}$ reveals specific associations with cognitive performance.
\end{abstract}

\section{Introduction}

White matter hyperintensities (WMHs) on $\mathrm{T}_{2}$-weighted magnetic resonance images (MRI) are radiological signs of cerebral small vessel disease (SVD) (Wardlaw et al., 2013). They are associated with a higher incidence of stroke and dementia (Debette and Markus, 2010), mood disorders, motor impairments and urinary incontinence (Poggesi et al., 2011). Moreover, WMHs are related to cognitive impairments, particularly executive dysfunctions and poorer psychomotor speed
(Bolandzadeh et al., 2012). Whilst WMHs have acquired considerable interest in the field of translational and clinical research, the assessment and the reporting of $\mathrm{WMH}$ volume are often inconsistent in research studies and medical practice (Frey et al., 2019).

The optimal MRI sequence to assess WMHs is fluid-attenuated inversion recovery (FLAIR). This sequence generates $\mathrm{T}_{2}$-weighted images where the signal from the cerebrospinal fluid is suppressed and hyperintense regions stand out on a low intensity homogeneous background (Wardlaw et al., 2013). In research, quantification of WMHs is

\footnotetext{
* Corresponding author at: Department of Biomedical Sciences for Health, Università degli Studi di Milano, Via Luigi Mangiagalli 31, 20133 Milano, Italy.

E-mail address: luca.melazzini@unimi.it (L. Melazzini).
} 
preferred to qualitative assessment due to higher reliability, sensitivity and objectivity of the former (De Guio et al., 2016; Van den Heuvel et al., 2006) and the widespread availability of segmentation software. However, the interpretation of quantitative results and comparison between studies remain difficult due to acquisition-related differences (scanner, protocol), discrepancies between processing methods (pre-processing pipelines, method/tool used to extract WMH measurements) and variations in the definition of what should be considered a WMH (De Guio et al., 2016). Harmonisation methods that reduce or compensate for the variability due to acquisition differences and/or processing discrepancies are being developed to enable comparisons between or pooling of MRI-derived measures from different datasets (Bordin et al., 2020). Notwithstanding, the lack of a clear definition on what should be segmented as a $\mathrm{WMH}$ and whether some $\mathrm{WMH}$ sub-classes are more clinically relevant than others warrant further investigation (Alber et al., 2019; Frey et al., 2019; Murray et al., 2010; Smith et al., 2019; Tate et al., 2008; Wardlaw et al., 2013).

Periventricular WMHs are more strongly associated with concurrent cognitive deficits than deep ones (Bolandzadeh et al., 2012). This is in line with longitudinal studies on regional baseline WMH volumes and their association with the risk of transition from intact cognition to mild cognitive impairment and dementia (De Groot et al., 2002; Kim et al., 2015; van Straaten et al., 2008). To explain this finding, the hypothesis of reduced brain reserve in periventricular regions has been put forward (De Groot et al., 2002). Despite this evidence, it is still unclear whether periventricular and deep WMHs would constitute a continuous entity or should be considered and reported separately (DeCarli et al., 2005). If the latter is true, a single method for distinguishing types of WMHs should be adopted among the many that have been proposed (Griffanti et al., 2018).

White matter hyperintensities can either be iso- or hypointense in $\mathrm{T}_{1}$ weighted images (Spilt et al., 2006; Wardlaw et al., 2013). To the best of our knowledge, there are no studies performed on brain tissue samples that investigate whether there is any difference in the underlying pathology between non $\mathrm{T}_{1}$-hypointense versus $\mathrm{T}_{1}$-hypointense $\mathrm{WMHs}$. However, clinical research in multiple sclerosis has showed that demyelinating $\mathrm{T}_{1}$-hypointense lesions ("black holes") represent permanent damage to the white matter and are associated with cognitive impairments (Nowaczyk et al., 2019; Ozakbas et al., 2019). In WMHs of presumed vascular origin, the co-located hypointensity in $\mathrm{T}_{1}$-weighted images (here on referred to as $\mathrm{T}_{1}$-hypointense WMHs) may indicate more severe damage to the white matter than WMHs that are visible in $\mathrm{T}_{2}$-weighted images without any corresponding hypointensities in the $\mathrm{T}_{1}$-weighted images (here on referred to as non $\mathrm{T}_{1}$-hypointense $\mathrm{WMHs}$ ). Quantitative measures of the motion of water molecules in vivo using diffusion tensor imaging (DTI) metrics have shown microstructural changes in the white matter in WMH areas (Soriano-Raya et al., 2014; Wardlaw et al., 2015). Altered DTI metrics were reported in multiple sclerosis lesions that turn into permanent "black holes" (Naismith et al., 2010). Similarly, we could expect lower fractional anisotropy and higher radial diffusivity to reflect more severe axonal and myelin damage in $\mathrm{T}_{1}$ hypointense WMHs than in non $\mathrm{T}_{1}$-hypointense WMHs. $\mathrm{T}_{1}$-hypointense WMHs could thereby represent the portion of WMHs that carries the highest clinical impact.

For the reasons outlined above, we tested the hypothesis that periventricular (rather than deep) and $\mathrm{T}_{1}$-hypointense (rather than non $\mathrm{T}_{1}$ hypointense) WMHs may indicate the most severe forms of lesion in terms of impact on cognitive function. To this purpose, we classified WMHs according to both their anatomical location and intensity in $\mathrm{T}_{1}$ weighted images in a large cohort of community-dwelling older adults, and studied whether this classification could provide added value on the association between WMHs and cognitive function. We implemented a method for categorizing WMHs according to spatial location and intensity in $\mathrm{T}_{1}$-weighted images that was automatic and objective and used multiple linear regression analysis to see if these WMH sub-classes show specific associations with validated scores of cognitive functions.
Given the current limitations and discrepancies in WMHs definition, our ultimate goal was to identify which sub-class(es) are specifically linked to cognitive function. This would inform future guidelines to focus the assessment on clinically-relevant radiological criteria of WMHs beyond their total extent, with a clear and objective definition of WMHs radiological appearance and location.

\section{Methods}

The study sample was drawn from 800 participants of the Whitehall II imaging sub-study (Filippini et al., 2014), which is part of a larger prospective occupational cohort study of British civil servants established in 1985 (Marmot and Brunner, 2005). Participants were between 60 and 85 years of age at the time the MRI study started. Participants with contraindications to MRI scanning or who were unable to travel to Oxford without assistance were excluded from the study. Ethical approval was obtained from the University of Oxford Central University Research Ethics Committee, and the UCL Medical School Committee on the Ethics of Human Research. Informed written consent was obtained from all participants. Socio-demographic, health and lifestyle variables and current cognitive function were assessed at the time of the MRI.

MRI data were acquired at the Oxford Centre for Functional MRI of the Brain (FMRIB), Wellcome Centre for Integrative Neuroimaging (University of Oxford), using a 3-T, Siemens Magnetom Verio (Erlangen, Germany) scanner with a 32-channel receive head coil from April 2012 to December 2014 ( $N=550$ participants) and a 3-T Siemens Prisma (Erlangen, Germany) with a 64-channel receive head-neck coil from July 2015 to December 2016 ( $N=250$ participants) due to a scanner upgrade. Details of acquisition protocols are shown in (Filippini et al., 2014) and (Zsoldos et al., 2020) and are reported in Supplementary Table S1. For the purpose of this study we used high-resolution $\mathrm{T}_{1}$ weighted images, FLAIR images and diffusion weighted images (DWI).

All images were processed and analysed using FMRIB Software Library (FSL) v.6.0 tools (Jenkinson et al., 2012). Participants' $\mathrm{T}_{1}$ weighted and FLAIR images were skull-stripped with FSL-BET (Smith, 2002) and bias field corrected with FSL-FAST (Zhang et al., 2001). DWI scans were pre-processed as described in (Filippini et al., 2014) and a diffusion tensor model was fit at each voxel to obtain maps of fractional anisotropy (FA), mean diffusivity (MD), axial diffusivity (AD) and radial diffusivity $(\mathrm{RD}) . \mathrm{T}_{1}$-weighted and FA images were then linearly registered to the corresponding FLAIR with FSL-FLIRT (Jenkinson and Smith, 2001). WMHs segmentation was performed with FSL-BIANCA (Griffanti et al., 2016), using intensity features ( $\mathrm{T}_{1}$-weighted and FLAIR), local average intensities (3 voxels kernel), and spatial features (MNI coordinates obtained from the transformation between FLAIR and MNI for each subject, weighting factor of 2). To avoid scanner-specific biases in the estimates, BIANCA was trained with WMH masks manually delineated in a sub-sample of individuals scanned on the Prisma $(n=24)$ and Verio ( $\mathrm{n}=24$ ) scanners and an independent sample from the UK Biobank study $(\mathrm{n}=12)$. The processing steps and the training settings have been previously optimised (Bordin et al., 2020) to offer the best balance between segmentation performance and removal of scanner-specific biases in the WMH estimates. The total WMH mask included voxels exceeding a probability of 0.9 of being a WMH and located within a white matter mask as described in (Griffanti et al., 2016). Finally, WMH masks were visually checked by an experienced observer to exclude images where small segmentation errors could hinder subsequent WMH sub-classification. The total WMH mask included voxels exceeding a probability of 0.9 of being a WMH and located within a white matter mask as described in (Griffanti et al., 2016). The total WMH volume was adjusted for the total brain volume and log transformed for statistical analysis.

We separated WMHs voxels into $\mathrm{T}_{1}$-hypointense and non $\mathrm{T}_{1}$-hypointense. To achieve this, we used FSL-FAST (Zhang et al., 2001) on $\mathrm{T}_{1}$ weighted images to perform tissue type segmentation and calculate maps of partial volume estimates (PVE) for the three classes (grey 
matter, white matter and cerebrospinal fluid). Due to their low-intensity values, $\mathrm{T}_{1}$-hypointense WMHs are classified by FAST as either grey matter or cerebrospinal fluid. We therefore classified voxels as non $\mathrm{T}_{1^{-}}$ hypointense WMHs the voxels within the total WMH mask where the corresponding white matter PVE was greater than 0.5 . We then obtained $\mathrm{T}_{1}$-hypointense WMHs by subtraction.

We used a cluster-based approach to separate between periventricular and deep WMHs, similar to the "continuity to ventricle" criterion described by (Griffanti et al., 2018). To do so, we created an extended ventricle mask (i.e. a ventricle mask that extended beyond the ventricular boundaries) in the Montreal Neurological Institute (MNI) space. The extended ventricle mask consisted of the probability maps -set with a very low threshold- of the lateral ventricles, thalami and fornix bilaterally. We transformed the mask to the single-subject FLAIR space via the corresponding $\mathrm{T}_{1}$ using linear (Jenkinson et al., 2002) and non-linear registration (Andersson et al., 2007), and classified as periventricular WMHs the clusters that overlapped with any part of the mask. Deep WMHs were then defined by subtraction.

We finally combined the two criteria and obtained four WMH masks for the following sub-classes for each participant: periventricular $\mathrm{T}_{1^{-}}$hypointense WMHs; periventricular non $\mathrm{T}_{1}$-hypointense WMHs; deep $\mathrm{T}_{1}$-hypointense WMHs; deep non $\mathrm{T}_{1}$-hypointense WMHs. Then, each mask was used to derive the corresponding WMH volume, which was adjusted for the total brain volume and log transformed for statistical analysis.

We performed univariate multiple linear regression using the univariate general linear model function type III sum of squares on SPSS version 25.0 (IBM Corp. Armonk, NY). The following measures of participants' cognition were selected as indices of global functioning, executive function, processing speed, working memory and word retrieval, in line with (Bolandzadeh et al., 2012): Montreal cognitive assessment (MoCA), trail-making test (TMT, A and B), digit span forward, backwards and sequence, digit symbol, digit coding, Boston naming-60 test (BNT), phonemic (letter) fluency (FLU-L) and semantic (category) fluency (FLU-C) tests. For details on the cognitive tests please refer to (Filippini et al., 2014). Demographic variables (age at the examination, sex, total years of education, systolic blood pressure, diastolic blood pressure) were used as covariates of no interest. For each cognitive test (dependent variable in the univariate multiple linear regression), two models were investigated, in which participants' demographic variables were kept unchanged while WMHs were included as either WMHs total volume (corresponding to the BIANCA output) or subdivided WMH volumes (corresponding to the four sub-classes reported above).

To better investigate the meaning of $\mathrm{T}_{1}$-hypointensity in our sample, we performed two post-hoc analyses. First, we studied WMH microstructure using DTI-derived metrics, given the lack of histopathological data for this dataset. Accordingly, we compared $\mathrm{T}_{1}$-hypointense and non $\mathrm{T}_{1}$-hypointense WMHs in terms of average fractional anisotropy (FA), mean diffusivity (MD), axial diffusivity (AD) and radial diffusivity (RD) using paired $t$-tests to evaluate potential differences in the underlying microstructure. Second, since we noticed that a WMH often includes both $\mathrm{T}_{1}$-hypointense and non $\mathrm{T}_{1}$-hypointense voxels, we adopted an alternative $\mathrm{T}_{1}$-weighted intensity-based classification of WMHs by dividing the total WMH map into $\mathrm{WMH}$ clusters with and without $\mathrm{T}_{1^{-}}$hypointense voxels. This sub-classification was then used to look at the prevalence of $\mathrm{WMH}$ clusters with a $\mathrm{T}_{1}$-hypointense component and to better interpret the results of the main analysis.

We also used Pearson correlations to further investigate the associations between WMH sub-classes, total WMH volume and age.

Statistical significance was set at $\alpha=0.05$. Further correction for multiple hypotheses testing was performed using Bonferroni correction (Di Leo and Sardanelli, 2020).

\section{Results}

Participants characteristics (demographics, cognitive scores and MRI measures) are reported in Table 1. One hundred and sixteen participants were excluded from our analysis due to incomplete or poor-quality images $(\mathrm{N}=44)$, neurological disorders $(\mathrm{N}=30)$ and inaccurate WMH segmentation masks $(\mathrm{N}=42)$ to leave a total of 684 communitydwelling older adults. Participants' age ranged from 60 to 83 years. Most of participants were male $(551 / 684,81 \%)$ and had a MoCA score greater than or equal to $26(551 / 684,81 \%)$.

An example of the WMH segmentation output resulting from the method we developed is provided in Fig. 1.

White matter hyperintensities expressed as total volume were not significantly associated with any of the cognitive tests. However, when the four sub-classes of WMHs were instead used in the model, we found statistically significant associations between periventricular $\mathrm{T}_{1}$-hypointense WMHs and poorer performance on the TMT-A $(\mathrm{p}=0.011)$, digit symbol $(\mathrm{p}=0.028)$ and digit coding $(\mathrm{p}=0.009)$ tests. Conversely, non $\mathrm{T}_{1}$-hypointense periventricular WMHs were associated with higher scores on the digit backwards test $(\mathrm{p}=0.023)$. Also, deep non $\mathrm{T}_{1}$ hypointense WMHs were found to be positively associated with the

Table 1

Sample characteristics.

\begin{tabular}{|c|c|c|c|c|c|}
\hline $\begin{array}{l}\text { Descriptive } \\
\text { statistics }\end{array}$ & $\mathrm{N}$ & Minimum & Maximum & Mean & $\begin{array}{l}\text { Standard } \\
\text { deviation }\end{array}$ \\
\hline \multicolumn{6}{|l|}{ Demographics } \\
\hline Age (years) & 684 & 60.34 & 83.03 & 69.65 & 5.06 \\
\hline Sex (M:F) & $551: 133$ & & & & \\
\hline $\begin{array}{l}\text { Total education } \\
\text { (years) }\end{array}$ & 684 & 13 & 28 & 19.10 & 2.85 \\
\hline $\begin{array}{l}\text { Systolic blood } \\
\text { pressure } \\
(\mathrm{mmHg})\end{array}$ & 681 & 93 & 226 & 141.09 & 17.54 \\
\hline $\begin{array}{l}\text { Diastolic blood } \\
\text { pressure } \\
(\mathrm{mmHg})\end{array}$ & 680 & 47 & 114 & 77.21 & 10.77 \\
\hline \multicolumn{6}{|l|}{ Cognitive scores } \\
\hline MoCA & 684 & 17 & 30 & 27.25 & 2.25 \\
\hline Trail making A & 682 & 13 & 125 & 30.98 & 11.73 \\
\hline Trail making B & 680 & 24 & 321 & 67.08 & 33.84 \\
\hline Trail making B-A & 680 & -8 & 253 & 36.25 & 28.73 \\
\hline $\begin{array}{l}\text { Digit span } \\
\text { forward }\end{array}$ & 683 & 5 & 16 & 11.08 & 2.28 \\
\hline $\begin{array}{l}\text { Digit span } \\
\text { backwards }\end{array}$ & 683 & 4 & 16 & 9.65 & 2.45 \\
\hline $\begin{array}{l}\text { Digit span } \\
\text { sequence }\end{array}$ & 683 & 0 & 16 & 10.05 & 2.48 \\
\hline Digit symbol & 683 & 13 & 46 & 30.78 & 5.75 \\
\hline Digit coding & 683 & 13 & 114 & 63.02 & 13.22 \\
\hline $\begin{array}{l}\text { Boston naming } \\
\text { test- } 60\end{array}$ & 684 & 15 & 60 & 57.38 & 4.66 \\
\hline Letter fluency & 684 & 3 & 31 & 15.78 & 4.64 \\
\hline Category fluency & 684 & 3 & 40 & 22.36 & 5.34 \\
\hline \multicolumn{6}{|l|}{ MRI measures } \\
\hline $\begin{array}{l}\text { Total brain } \\
\quad \text { volume }\left(\mathrm{dm}^{3}\right)\end{array}$ & 684 & 1.023 & 1.928 & 1.456 & 0.133 \\
\hline $\begin{array}{l}\text { Total WMH } \\
\text { volume }\left(\mathrm{cm}^{3}\right)\end{array}$ & 684 & 0.339 & 35.627 & 5.855 & 3.715 \\
\hline $\begin{array}{l}\text { Periventricular } \\
\mathrm{T}_{1} \text {-hypointense } \\
\text { WMHs }\left(\mathrm{cm}^{3}\right)\end{array}$ & 684 & 0.240 & 31.448 & 2.679 & 3.025 \\
\hline $\begin{array}{l}\text { Periventricular } \\
\text { non } \mathrm{T}_{1^{-}} \\
\text {hypointense } \\
\text { WMHs }\left(\mathrm{cm}^{3}\right)\end{array}$ & 684 & 0.050 & 6.379 & 2.138 & 0.831 \\
\hline $\begin{array}{l}\text { Deep } \mathrm{T}_{1-} \\
\text { hypointense } \\
\text { WMHs }\left(\mathrm{cm}^{3}\right)\end{array}$ & 684 & 0.001 & 5.274 & 0.434 & 0.577 \\
\hline $\begin{array}{l}\text { Deep non } \mathrm{T}_{1^{-}} \\
\text {hypointense } \\
\text { WMHs }\left(\mathrm{cm}^{3}\right)\end{array}$ & 684 & 0.003 & 3.976 & 0.604 & 0.565 \\
\hline
\end{tabular}

Legend: MoCA, Montreal cognitive assessment; WMHs, white matter hyperintensities. 


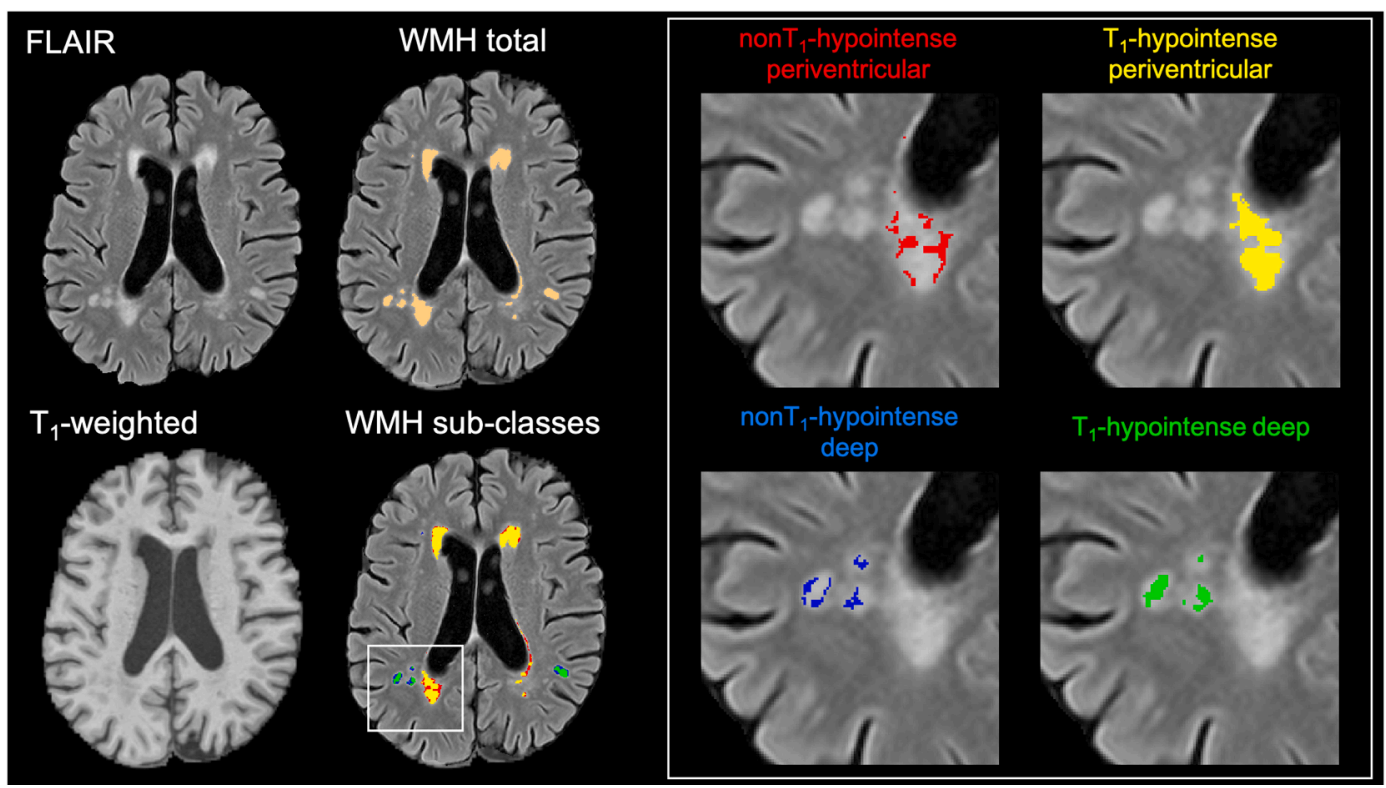

Fig. 1. The left panel shows the native images (FLAIR and $\mathrm{T}_{1}$-weighted) and image processing outputs (WMH total and WMH sub-classes) for a subject drawn from the study sample. The right panel shows a colour-coded detailed view of the four sub-classes of WMHs.

letter $(\mathrm{p}=0.004)$ and category $(\mathrm{p}=0.036)$ fluency tests. Regression analysis results for the models where WMHs sub-classes were significantly associated with cognitive performances are shown in Table 2. Comprehensive results for all the models, including all covariates, are showed in Supplementary Table S2.

When looking at microstructural differences in WMHs classified according to the intensity in $\mathrm{T}_{1}$-weighted images criterion, $\mathrm{T}_{1}$-hypointense WMHs showed significantly lower FA, and higher MD, AD and RD than non $\mathrm{T}_{1}$-hypointense WMHs ( $\mathrm{p}<0.001$ for all measures). Results of the within-subject paired $t$-tests for the averages of DTI-derived metrics between $\mathrm{T}_{1}$ - and non $\mathrm{T}_{1}$-hypointense WMHs are reported in Table 3.

Results for the separation of the total WMH volume into WMH clusters with and without corresponding $\mathrm{T}_{1}$-hypointense voxels are reported in Table 4.

We found that $50 \%$ of the WMH clusters had $\mathrm{T}_{1}$-hypointense voxels. However, despite constituting half of all clusters, these WMH clusters with at least one T1-hypointense voxel composed $94 \%$ of the whole $\mathrm{WMH}$ volume. The remaining $6 \%$ of the $\mathrm{WMH}$ volume consisted of small non $\mathrm{T}_{1}$-hypointense $\mathrm{WMH}$ clusters, i.e. WMH clusters without co-located
Table 3

Comparison between the $\mathrm{T}_{1}$ - and non $\mathrm{T}_{1}$ - hypointense WMHs in terms of DTIderived metrics. Paired $t$-tests results. Diffusivity values are expressed in $\left(\mathrm{mm}^{2} / \mathrm{s}\right) \times 10^{-3}$.

\begin{tabular}{|c|c|c|c|c|c|c|}
\hline \multirow[t]{2}{*}{ Metric } & \multicolumn{2}{|c|}{$\begin{array}{l}\mathrm{T}_{1} \text {-hypointense } \\
\text { WMHs }\end{array}$} & \multicolumn{2}{|c|}{$\begin{array}{l}\text { Non } T_{1} \text {-hypointense } \\
\text { WMHs }\end{array}$} & \multirow[t]{2}{*}{$t$} & \multirow[t]{2}{*}{ p-value } \\
\hline & Mean & SD & Mean & SD & & \\
\hline FA & 0.26 & 0.03 & 0.37 & 0.04 & -86.21 & $<0.001$ \\
\hline MD & 1.12 & 0.09 & 0.92 & 0.06 & 71.24 & $<0.001$ \\
\hline $\mathrm{AD}$ & 1.42 & 0.11 & 1.30 & 0.08 & 33.03 & $<0.001$ \\
\hline $\mathrm{RD}$ & 0.97 & 0.09 & 0.73 & 0.06 & 88.14 & $<0.001$ \\
\hline
\end{tabular}

Legend: FA, fractional anisotropy; MD, mean diffusivity; AD, axial diffusivity; $\mathrm{RD}$, radial diffusivity; SD, standard deviation; WMHs, white matter hyperintensities.

note: ${ }^{\#} \mathrm{P}$-values remained significant after adjustment for multiple comparisons across metrics.

Table 2

Multiple linear regression analysis results. Model results and significant WMH predictors of cognitive scores adjusted for age at the examination, sex, total years of education, systolic blood pressure and diastolic blood pressure.

\begin{tabular}{|c|c|c|c|c|c|c|}
\hline \multirow[b]{2}{*}{ Cognitive test } & \multicolumn{3}{|l|}{ Model } & \multicolumn{3}{|l|}{ Parameter estimates } \\
\hline & $\mathrm{F}$ & p-value & Adjusted $\mathrm{R}^{2}$ & Significant WMH covariate & $\beta$ (SE) & p-value ${ }^{\#}$ \\
\hline Trail making A & 10.08 & $<0.001$ & 0.11 & $\mathrm{~T}_{1}$-hypointense periventricular WMHs & $4.79(1.87)$ & 0.011 \\
\hline Digit span backwards & 5.92 & $<0.001$ & 0.06 & Non $T_{1}$-hypointense periventricular WMHs & $1.30(0.57)$ & 0.023 \\
\hline Digit span sequence & 4.99 & $<0.001$ & 0.05 & $\mathrm{~T}_{1}$-hypointense periventricular WMHs & $-0.79(0.41)$ & $0.054^{\wedge}$ \\
\hline Digit symbol & 8.25 & $<0.001$ & 0.09 & $\mathrm{~T}_{1}$-hypointense periventricular WMHs & $-2.05(0.93)$ & 0.028 \\
\hline Digit coding & 11.06 & $<0.001$ & 0.12 & $\mathrm{~T}_{1}$-hypointense periventricular WMHs & $-5.52(2.09)$ & 0.009 \\
\hline Letter fluency & 3.74 & $<0.001$ & 0.04 & Non $\mathrm{T}_{1}$-hypointense deep WMHs & $2.10(0.72)$ & 0.004 \\
\hline Category fluency & 8.07 & $<0.001$ & 0.09 & Non $T_{1}$-hypointense deep WMHs & $1.83(0.87)$ & 0.036 \\
\hline
\end{tabular}

Legend: SE, standard error; WMHs, white matter hyperintensities.

note: ${ }^{\#} \mathrm{P}$-values did not remain significant after adjustment for multiple comparisons across cognitive tests.

note: ${ }^{\wedge}$ Predictor that shows a trend for significance $(0.05<\mathrm{p}<0.06)$. 
Table 4

Number and size of WMH clusters with and without $\mathrm{T}_{1}$-hypointense voxels.

\begin{tabular}{|c|c|c|c|}
\hline & Number & $\begin{array}{l}\text { Average volume } \\
\text { per subject }\left(\mathrm{cm}^{3}\right)\end{array}$ & $\begin{array}{l}\text { Average volume } \\
\text { per cluster }\left(\mathrm{mm}^{3}\right)\end{array}$ \\
\hline WMH clusters & $\begin{array}{l}223.91 \pm \\
103.77\end{array}$ & $5.86 \pm 3.72$ & $36 \pm 42$ \\
\hline $\begin{array}{l}\text { Clusters without } \mathrm{T}_{1^{-}} \\
\text {hypointense voxels }\end{array}$ & $\begin{array}{l}112.43 \pm \\
59.43\end{array}$ & $0.30 \pm 0.19$ & $3 \pm 1$ \\
\hline $\begin{array}{l}\text { Clusters with } \mathrm{T}_{1^{-}} \\
\text {hypointense voxels }\end{array}$ & $\begin{array}{l}111.48 \pm \\
57.55\end{array}$ & $5.56 \pm 3.75$ & $66 \pm 63$ \\
\hline Rims & & $2.45 \pm 1.02$ & $28 \pm 19$ \\
\hline Cores & & $3.11 \pm 3.23$ & $38 \pm 50$ \\
\hline
\end{tabular}

Legend: WMHs, white matter hyperintensities.

hypointensity in $\mathrm{T}_{1}$-weighted images.

The volume of non $\mathrm{T}_{1}$-hypointense WMHs is the sum of non $\mathrm{T}_{1^{-}}$ hypointense clusters and the hyperintense rims of $\mathrm{T}_{1}$-hypointense clusters (a schematic representation is depicted in Fig. 2).

Given the unexpected significant positive associations of non $\mathrm{T}_{1^{-}}$ hypointense WMHs with the digit span backwards, letter and category fluency, we investigated which of these two components (either non $\mathrm{T}_{1^{-}}$ hypointense clusters or rims of $\mathrm{T}_{1}$-hypointense clusters) drove the observed associations. When fitting multiple linear regression models with either of these two components as the only WMH predictor for the fluency and digit span backwards scores, we found that non $\mathrm{T}_{1}$-hypointense clusters were significantly associated with the fluency performances (FLU-L: $\beta=1.98, p=0.001$; FLU-C: $\beta=2.02, p=0.004$ ). Conversely, rims were significant predictors of the digit span backwards scores $(\beta=0.92, \mathrm{p}=0.035)$.

Additional multiple regression models and Pearson correlations among WMH sub-classes, total WMH volume and age supporting these findings are reported in Supplementary Tables S3 and S4.

\section{Discussion}

In this study we sought to provide a clinically-oriented insight into WMHs by developing an automated method for classifying WMHs according to spatial location (periventricular versus deep WMHs) and lesion intensity in the corresponding $\mathrm{T}_{1}$-weighted image $\left(\mathrm{T}_{1}\right.$-hypointense versus non $\mathrm{T}_{1}$-hypointense $\mathrm{WMHs}$ ). We fitted univariate multiple linear regression models using the volumes of WMH sub-classes as predictors for the participants' performance in several cognitive tests and then further explored the microstructural properties of $\mathrm{T}_{1}$-hypointense WMHs to understand the meaning of this radiological appearance. Our classification proved to be clinically meaningful, as periventricular $\mathrm{T}_{1}$-hypointense WMHs were found to be linked to poorer performance on multiple cognitive tests, including the trail making A, digit symbol and digit coding tests. Since most of participants showed global functioning scores within normal limits, our sub-classification method could predominantly prove useful in healthy elderly. Subjects without overt cognitive decline or dementia would also benefit more from prompt preventive and therapeutic interventions. Overall, our results suggest that sub-classifying WMHs according to intensity and spatial location may turn out to be useful for investigating cognitive performance in the ageing population. Notably, periventricular T1hypointense WMHs would represent a promising WMH biomarker for investigating cognitive domains related to executive function and processing speed.

Our finding of periventricular WMHs being associated with poorer executive function and psychomotor speed scores is in line with previous large longitudinal population-based studies on non-demented elderly (De Groot et al., 2002; Griffanti et al., 2018), where periventricular WMHs were found to be more strongly associated with participants' cognition than deep WMHs. Increased volume of periventricular WMHs in the Alzheimer Disease Neuroimaging Initiative dataset was also associated with evidence of beta-amyloid deposition in the brain
(Marnane et al., 2016), suggesting a synergistic damage driven by concurrent SVD and Alzheimer's pathology in these areas. Periventricular regions are also characterised by high density of long associating fibres which link the cortex to the deep grey matter and other distant brain territories (Filley, 1998). For this reason, they are potentially susceptible to pathologies that damage cortical arteries and eventually provoke distal hypoperfusion (Moody et al., 1990). Disrupted cholinergic activity is related to periventricular (and not deep) WMHs and may be involved in the physio-pathological pathway that underpins the observed cognitive scores (Bohnen et al., 2009). Moreover, high periventricular WMHs were found to be associated with frontal cortical thinning, where both imaging findings were independently linked to executive dysfunctions (Seo et al., 2012). Altogether, these findings endorse our results of periventricular WMHs being more strongly associated with domain-specific poorer cognitive performance than deep WMHs.

Although the location criterion is well established, less is known about the meaning of $\mathrm{T}_{1}$-intensity in WMHs. This aspect has been wellinvestigated in demyelinating disease where hypointense lesions in $\mathrm{T}_{1^{-}}$ weighted images are more likely to represent low axonal density and irreversible tissue damage (Bitsch et al., 2001; Van Walderveen et al., 1998). Our DTI analysis showed decreased FA and increased MD, AD and $\mathrm{RD}$ in $\mathrm{T}_{1}$-hypointense WMHs. Not surprisingly, these findings mirror similar microstructural results found in multiple sclerosis (Vavasour et al., 2019) and suggest more severe damage to the white matter in $T_{1}$ hypointense areas than their $\mathrm{T}_{1}$-isointense counterpart.

$\mathrm{T}_{1}$-hypointense and non $\mathrm{T}_{1}$-hypointense WMHs may represent two distinct entities with different meanings. Alternatively, WMHs could start as small punctate FLAIR hyperintensities and later develop a $\mathrm{T}_{1^{-}}$ hypointense "core". Despite the lack of longitudinal data in our cohort, we attempted to investigate further the meaning of the intensity in $\mathrm{T}_{1^{-}}$ weighted images and the theoretical evolution of WMHs and their intensity in $\mathrm{T}_{1}$-weighted images from a cross-sectional basis. Within the total WMH mask, we separated WMH clusters that contained some $\mathrm{T}_{1}$ hypointense voxels ( $\mathrm{T}_{1}$-hypointense clusters) from those that did not (non $\mathrm{T}_{1}$-hypointense clusters) (Table 4). Since most of the $\mathrm{WMH}$ volume comprised (relatively big) clusters with a $\mathrm{T}_{1}$-hypointense component, our results suggest that the evolution of a WMH may be a cascade of events starting from small punctate lesions leading to bigger lesions with a $\mathrm{T}_{1}$-hypointense core and surrounding non $\mathrm{T}_{1}$-hypointense rim (Fig. 2).

This mechanism could explain the significant association we observed between higher volume of deep non $\mathrm{T}_{1}$-hypointense WMHs and higher scores at the fluency tests. Since the volume of non $\mathrm{T}_{1}$ hypointense WMHs includes both the volume of non $\mathrm{T}_{1}$-hypointense clusters and the outer rim of the clusters with a $\mathrm{T}_{1}$-hypointense core, we hypothesized that the positive association of deep non $\mathrm{T}_{1}$-hypointense WMHs with higher cognitive scores at the fluency tests could be driven by non- $\mathrm{T}_{1}$ hypointense clusters (i.e. smaller, less severe WMHs, that have not yet progressed into a $\mathrm{WMH}$ with a $\mathrm{T}_{1}$-hypointense component). We tested this hypothesis by fitting multiple linear regression models with either the volume of all non $\mathrm{T}_{1}$-hypointense clusters or only the volume of deep non $\mathrm{T}_{1}$-hypointense WMHs voxels as the only imaging predictors for the fluency tests (Supplementary Table S3). Notably, non $\mathrm{T}_{1}$-hypointense clusters were more strongly associated with fluency scores than deep non $\mathrm{T}_{1}$-hypointense WMHs. Thus, it is likely that the volume of clusters of non $\mathrm{T}_{1}$-hypointense $\mathrm{WMHs}$ drove the observed significance of deep non $\mathrm{T}_{1}$-hypointense WMHs as predictors of better cognitive scores at the fluency tests.

The positive associations of non $\mathrm{T}_{1}$-hypointense clusters with fluency scores could be due to the fact that these small lesions are more frequent in younger individuals with a low WMH volume. The hypothesized evolution into $\mathrm{T}_{1}$-hypointense clusters would then explain the apparent decrease of this type of lesions in individuals with lower fluency scores.

Since non $\mathrm{T}_{1}$-hypointense clusters are small, we cannot exclude the possibility that some of these are false positives from BIANCA segmentation. However, WMH masks have been visually checked so that it is 
SIZE, (TIME)

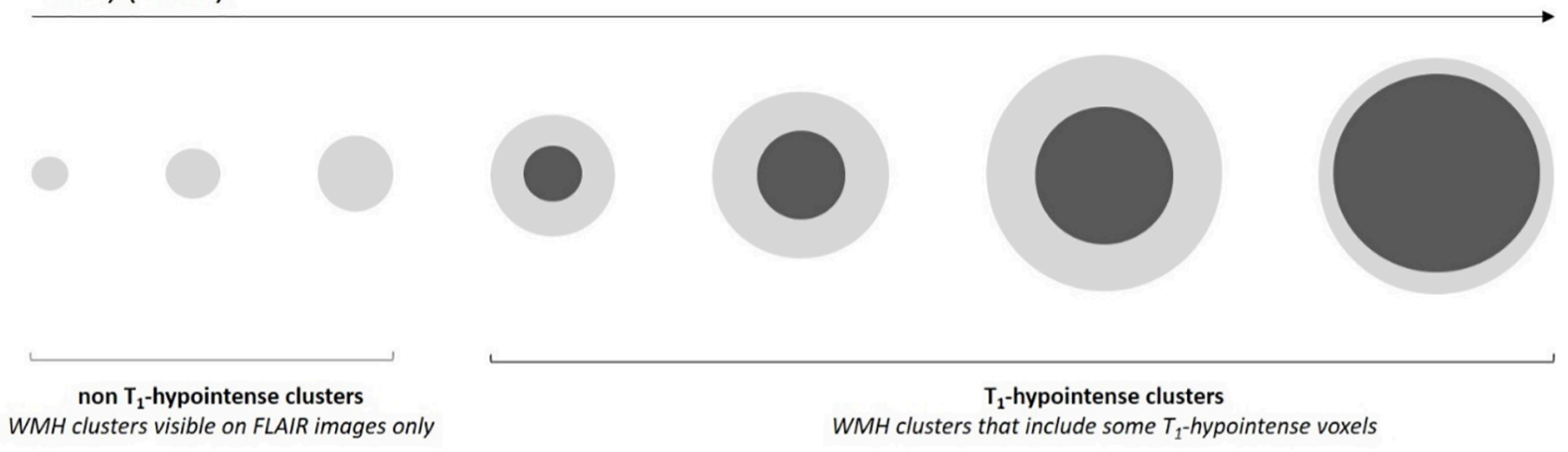

Fig. 2. Diagram representing the potential evolution of WMHs over time. WMHs, white matter hyperintensity.

unlikely that the results could be driven by errors in WMHs segmentation.

Our finding of higher non $\mathrm{T}_{1}$-hypointense periventricular WMHs linked to higher scores at the digit span backwards test could instead be explained by the other component of non $\mathrm{T}_{1}$-hypointense WMHs, the non $\mathrm{T}_{1}$-hypointense voxels belonging to $\mathrm{T}_{1}$-hypointense clusters (i.e. the hyperintense "rims"). In fact, when fitting the multiple linear regression model with the volumes of rims of all $\mathrm{T}_{1}$-hypointense clusters as the only imaging predictor, we found a positive association with the digit span backwards that was very similar to the one given by non $\mathrm{T}_{1}$-hypointense periventricular WMHs alone (Supplementary Table S3). Although rim and core belong to the same physical entity (i.e. the WMH cluster) they are likely to have opposite meanings. On the one hand, $\mathrm{T}_{1}$-hypointense WMHs voxels predict bad cognitive scores, as seen for the Trail Making Test A, digit symbol and digit coding tests. On the other hand, in spite of the positive association between rims and cores in terms of overall volume, the former are predictors of higher cognitive scores from the digit span backwards test. Thus, within the context of our hypothesized evolution of WMHs, rims would represent those WMH areas belonging to $\mathrm{T}_{1}$-hypointense clusters that have not turned $\mathrm{T}_{1}$-hypointense yet and theoretically "withstand" further tissue damage. When this occurs, the number of non $T_{1}$-hypointense voxels would decrease because they become $\mathrm{T}_{1}$-hypointense. This would in turn explain the positive relationship with cognition observed in our results. Further investigation would be necessary to explore the potential cascade of events leading to change in $\mathrm{T}_{1}$ intensity in a longitudinal setting, since the hypothesis of rims as WMHs associated with healthy cognitive aging is very speculative.

Our study has some limitations in terms of the data and the methodology.

The Whitehall II imaging sub-study dataset shows a narrow age range (60-84 years) and a strong gender imbalance, skewed towards men, since it reflects the demographic of British civil servants at the time of recruitment in the main study. Moreover, our finding that higher periventricular WMHs are linked to poorer cognitive scores is based on the definition of periventricular WMHs as the WMHs that are contiguous with the margins of each lateral ventricle. Despite previous comparisons of different ways to define periventricular WMHs on this population giving comparable results across criteria (Griffanti et al., 2018), we cannot exclude that other sub-classification criteria may yield different results. Future work is therefore needed to test the generalisability of our findings. Furthermore, our approach for the sub-classification of WMHs relies on automated segmentation of WMHs with BIANCA, tissue type segmentation with FAST, and registration of images with different spatial resolutions. As already mentioned, we cannot exclude inaccuracies in the WMH masks, despite visual inspection of the results. We used FAST segmentation as a proxy for defining $\mathrm{T}_{1}$-hypointensity, therefore inaccuracies in the segmentation would translate to inaccuracies in the sub-classification. Moreover, we performed linear and non- linear registrations between images with different resolutions (FLAIR, $\mathrm{T}_{1}$ and MNI space) and the interpolation process could have slightly affected the segmented volumes.

Finally, our hypothesis on the evolution of WMHs should be interpreted cautiously and prompt further longitudinal studies. For example, it would be very valuable to follow up participants and study how WMH sub-classes evolve over time to validate the proposed theory. Another interesting future development would be looking at how the different WMH sub-classes are related to incidence of diseases, such as stroke and dementia, using risk models in well-balanced longitudinal datasets. If results are confirmed, our classification system could ultimately be translated into the clinic.

Despite these limitations, this study presents some novel theoretical and methodological insights that can contribute to better understanding of the role of WMHs in cognitive aging. The methods developed herein can be easily adopted in other research settings. The extended ventricle mask and the scripts created for images post-processing are publicly available $^{1}$. These scripts can also be equally applied to any manually- or automatically-derived WMH masks, other than those from BIANCA, to obtain the four WMH sub-classes presented in this study.

\subsection{Conclusion}

We showed that information from spatial location and intensity in $\mathrm{T}_{1}$-weighted images provide potentially clinically useful insights into the meaning of WMHs with regards to participants' cognitive function. Notably, the combination of these two criteria revealed an association with cognitive scores related to executive function, processing speed, working memory and language, that the WMH total volume alone could not provide.

\section{CRediT authorship contribution statement}

Luca Melazzini: Conceptualization, Formal analysis, Investigation, Data curation, Methodology, Software, Visualization, Writing - original draft. Clare E. Mackay: Conceptualization, Funding acquisition, Investigation, Methodology, Supervision, Visualization, Writing - review \& editing. Valentina Bordin: Investigation, Methodology, Software, Writing - review \& editing. Sana Suri: Investigation, Writing review \& editing. Eniko Zsoldos: Data curation, Project administration, Investigation, Visualization, Writing - review \& editing. Nicola Filippini: Data curation, Investigation, Project administration, Writing review \& editing. Abda Mahmood: Data curation, Project administration, Writing - review \& editing. Vaanathi Sundaresan: Methodology, Software, Writing - review \& editing. Marina Codari: Supervision, Writing - review \& editing. Eugene Duff: Investigation, Methodology,

\footnotetext{
${ }^{1}$ Git repository: https://git.fmrib.ox.ac.uk/ludovica/wmh-sub-classes
} 
Writing - review \& editing. Archana Singh-Manoux: Funding acquisition, Project administration, Writing - review \& editing. Mika Kivimäki: Funding acquisition, Project administration, Writing - review \& editing. Klaus P. Ebmeier: Data curation, Funding acquisition, Investigation, Methodology, Project administration, Supervision, Writing - review \& editing. Mark Jenkinson: Conceptualization, Investigation, Methodology, Software, Supervision, Writing - review \& editing. Francesco Sardanelli: Methodology, Supervision, Writing review \& editing. Ludovica Griffanti: Conceptualization, Funding acquisition, Investigation, Data curation, Methodology, Project administration, Software, Supervision, Visualization, Writing - original draft.

\section{Declaration of Competing Interest}

The authors declare that they have no known competing financial interests or personal relationships that could have appeared to influence the work reported in this paper.

\section{Acknowledgements}

We thank all Whitehall II participants for their time, the Whitehall II staff at the University College London, Mandy Pipkin and Barbora Krausova for assisting with recruitment and data collection, the FMRIB Radiographers team for data acquisition, IT and support teams at the Wellcome Centre for Integrative Neuroimaging for their helpful collaboration. The study follows MRC data sharing policies (https:// www.mrc.ac.uk/research/policies-and-guidance-for-researchers/datasharing/). Data will be accessible via the Dementias Platform UK (https://portal.dementiasplatform.uk/) after 2020.

\section{Funding}

The study was supported by the UK Medical Research Council (MRC) grants "Dementias Platform UK" (MR/L023784/2) and "Predicting MRI abnormalities with longitudinal data of the Whitehall II Substudy" (UK Medical Research Council: G1001354, PI: KPE), and by the HDH Wills 1965 Charitable Trust (Nr: 1117747, PI: KPE). This study was also supported by the Wellcome Centre for Integrative Neuroimaging, which has core funding from the Wellcome Trust (203139/Z/16/Z).

C.E.M., N.F. and L.G. were supported by the National Institute for Health Research (NIHR) Oxford Health Biomedical Research Centres (BRC), a partnership between Oxford Health NHS Foundation Trust and the University of Oxford. L.G. was also supported by the Oxford Parkinson's Disease Centre (Parkinson's UK Monument Discovery Award, J1403) and the MRC Dementias Platform UK. E.Zs, K.P.E. and S.S. were supported by the European Union's Horizon 2020 programme 'Lifebrain' (732592). S.S. was also supported by an Alzheimer's Society Junior Research Fellowship (Grant ref: 441). V.S. and M.J. were supported by the Wellcome Centre for Integrative Neuroimaging, which has core funding from the Wellcome Trust (203139/Z/16/Z). M.J. was supported by the National Institute for Health Research (NIHR) Oxford Biomedical Research Centre (BRC), and this research was funded by the Wellcome Trust (215573/Z/19/Z). A.S.-M. receives research support from the US National Institutes of Health (R01AG056477). M.K. was supported by NordForsk, the UK Medical Research Council (MRC S011676), the Academy of Finland (311492), and the US National Institutes on Aging (NIA R01AG056477, RF1AG062553).

\section{Appendix A. Supplementary data}

Supplementary data to this article can be found online at https://doi. org/10.1016/j.nicl.2021.102616.

\section{References}

Alber, J., Alladi, S., Bae, H., Barton, D.A., Beckett, L.A., Bell, J.M., Berman, S.E., Biessels, G.J., Black, S.E., Bos, I., Bowman, G.L., Brai, E., Brickman, A.M., Callahan, B.L., Corriveau, R.A., Fossati, S., Gottesman, R.F., Gustafson, D.R., Hachinski, V., Hayden, K.M., Helman, A.M., Hughes, T.M., Isaacs, J.D., Jefferson, A. L., Johnson, S.C., Kapasi, A., Kern, S., Kwon, J.C., Kukolja, J., Lee, A., Lockhart, S.N., Murray, A., Osborn, K.E., Power, M.C., Price, B.R., Rhodius-Meester, H.F.M., Rondeau, J.A., Rosen, A.C., Rosene, D.L., Schneider, J.A., Scholtzova, H., Shaaban, C.E., Silva, N.C.B.S., Snyder, H.M., Swardfager, W., Troen, A.M., Veluw, S. J., Vemuri, P., Wallin, A., Wellington, C., Wilcock, D.M., Xie, S.X., Hainsworth, A.H., 2019. White matter hyperintensities in vascular contributions to cognitive impairment and dementia (VCID): knowledge gaps and opportunities. Alzheimer's Dement. Transl. Res. Clin. Interv. 5, 107-117. https://doi.org/10.1016/j. trci.2019.02.001.

Andersson, J.L.R., Jenkinson, M., Smith, S., 2007. Non-linear registration, aka spatial normalisation. FMRIB Tech. Rep. TR07JA2 22.

Bitsch, A., Kuhlmann, T., Stadelmann, C., Lassmann, H., Lucchinetti, C., Brück, W., 2001. A longitudinal MRI study of histopathologically defined hypointense multiple sclerosis lesions. Ann. Neurol Off. J. Am. Neurol. Assoc. Child Neurol. Soc. 49, 793-796.

Bohnen, N.I., Mueller, M.L.T.M., Kuwabara, H., Constantine, G.M., Studenski, S.A., 2009. Age-associated leukoaraiosis and cortical cholinergic deafferentation. Neurology 72 , 1411-1416.

Bolandzadeh, N., Davis, J.C., Tam, R., Handy, T.C., Liu-Ambrose, T., 2012. The association between cognitive function and white matter lesion location in older adults: a systematic review. BMC Neurol. 12, 126.

Bordin, V., Bertani, I., Mattioli, I., Sundaresan, V., McCarthy, P., Suri, S., Zsoldos, E., Filippini, N., Mahmood, A., Melazzini, L., 2020. Integrating large-scale neuroimaging research datasets: harmonisation of white matter hyperintensity measurements across Whitehall and UK Biobank datasets. bioRxiv.

De Groot, J.C., De Leeuw, F., Oudkerk, M., Van Gijn, J., Hofman, A., Jolles, J., Breteler, M.M.B., 2002. Periventricular cerebral white matter lesions predict rate of cognitive decline. Ann. Neurol. 52, 335-341.

De Guio, F., Jouvent, E., Biessels, G.J., Black, S.E., Brayne, C., Chen, C., Cordonnier, C., De Leeuw, F.-E., Dichgans, M., Doubal, F., 2016. Reproducibility and variability of quantitative magnetic resonance imaging markers in cerebral small vessel disease. J. Cereb. Blood Flow Metab. 36, 1319-1337.

Debette, S., Markus, H.S., 2010. The clinical importance of white matter hyperintensities on brain magnetic resonance imaging: systematic review and meta-analysis. BMJ 341, c 3666.

DeCarli, C., Fletcher, E., Ramey, V., Harvey, D., Jagust, W.J., 2005. Anatomical mapping of white matter hyperintensities (wmh) exploring the relationships between periventricular WMH, deep WMH, and total WMH burden. Stroke 36, 50-55.

Di Leo, G., Sardanelli, F., 2020. Statistical significance: p value, 0.05 threshold, and applications to radiomics - reasons for a conservative approach. Eur. Radiol. Exp. 4, $1-8$.

Filippini, N., Zsoldos, E., Haapakoski, R., Sexton, C.E., Mahmood, A., Allan, C.L., Topiwala, A., Valkanova, V., Brunner, E.J., Shipley, M.J., 2014. Study protocol: the Whitehall II imaging sub-study. BMC Psychiatr. 14, 1-16.

Filley, C.M., 1998. The behavioral neurology of cerebral white matter. Neurology 50, 1535-1540.

Frey, B.M., Petersen, M., Mayer, C., Schulz, M., Cheng, B., Thomalla, G., 2019. Characterization of white matter hyperintensities in large-scale MRI-studies. Front. Neurol. 10, 238.

Griffanti, L., Jenkinson, M., Suri, S., Zsoldos, E., Mahmood, A., Filippini, N., Sexton, C.E., Topiwala, A., Allan, C., Kivimäki, M., 2018. Classification and characterization of periventricular and deep white matter hyperintensities on MRI: a study in older adults. Neuroimage 170, 174-181.

Griffanti, L., Zamboni, G., Khan, A., Li, L., Bonifacio, G., Sundaresan, V., Schulz, U.G. Kuker, W., Battaglini, M., Rothwell, P.M., 2016. BIANCA (Brain Intensity AbNormality Classification Algorithm): a new tool for automated segmentation of white matter hyperintensities. Neuroimage 141, 191-205.

Jenkinson, M., Bannister, P., Brady, M., Smith, S., 2002. Improved optimization for the robust and accurate linear registration and motion correction of brain images. Neuroimage 17, 825-841.

Jenkinson, M., Beckmann, C.F., Behrens, T.E.J., Woolrich, M.W., Smith, S.M., 2012. Fsl. Neuroimage 62, 782-790.

Jenkinson, M., Smith, S., 2001. A global optimisation method for robust affine registration of brain images. Med. Image Anal. 5, 143-156.

Kim, S., Choi, S.H., Lee, Y.M., Kim, M.J., Kim, Y.D., Kim, J.Y., Park, J.H., Myung, W., Na, H.R., Han, H.J., 2015. Periventricular white matter hyperintensities and the risk of dementia: a CREDOS study. Int. Psychogeriatr. 27, 2069.

Marmot, M., Brunner, E., 2005. Cohort profile: the Whitehall II study. Int. J. Epidemiol. $34,251-256$.

Marnane, M., Al-Jawadi, O.O., Mortazavi, S., Pogorzelec, K.J., Wang, B.W., Feldman, H. H., Hsiung, G.-Y.-R., Initiative, A.D.N., 2016. Periventricular hyperintensities are associated with elevated cerebral amyloid. Neurology 86, 535-543.

Moody, D.M., Bell, M.A., Challa, V.R., 1990. Features of the cerebral vascular pattern that predict vulnerability to perfusion or oxygenation deficiency: an anatomic study. Am. J. Neuroradiol. 11, 431-439.

Murray, M.E., Senjem, M.L., Petersen, R.C., Hollman, J.H., Preboske, G.M., Weigand, S. D., Knopman, D.S., Ferman, T.J., Dickson, D.W., Jack, C.R., 2010. Functional impact of white matter hyperintensities in cognitively normal elderly subjects. Arch. Neurol. 67, 1379-1385. https://doi.org/10.1001/archneurol.2010.280. 
Naismith, R.T., Xu, J., Tutlam, N.T., Scully, P.T., Trinkaus, K., Snyder, A.Z., Song, S.-K., Cross, A.H., 2010. Increased diffusivity in acute multiple sclerosis lesions predicts risk of black hole. Neurology 74, 1694-1701.

Nowaczyk, N., Kalinowska-Łyszczarz, A., Paprzycki, W., Michalak, S., Kaźmierski, R., Pawlak, M.A., 2019. Spatial distribution of white matter degenerative lesions and cognitive dysfunction in relapsing-remitting multiple sclerosis patients. Neurol. Neurochir. Pol. 53, 18-25.

Ozakbas, S., Kahraman, T., Ozdogar, A.T., Abasiyanik, Z., Sagici, O., Yigit, P., 2019. Black holes in relation to cognitive and physical functions in persons with multiple sclerosis. In: 2019 Annual Meeting of the Consortium of Multiple Sclerosis Centers, CMSC.

Poggesi, A., Pantoni, L., Inzitari, D., Fazekas, F., Ferro, J., O’Brien, J., Hennerici, M., Scheltens, P., Erkinjuntti, T., Visser, M., 2011. 2001-2011: a decade of the LADIS (Leukoaraiosis And DISability) Study: what have we learned about white matter changes and small-vessel disease? Cerebrovasc. Dis. 32, 577.

Seo, S.W., Lee, J.-M., Im, K., Park, J.-S., Kim, S.-H., Kim, S.T., Ahn, H.-J., Chin, J. Cheong, H.-K., Weiner, M.W., 2012. Cortical thinning related to periventricular and deep white matter hyperintensities. Neurobiol. Aging 33, 1156-1167.

Smith, E.E., Biessels, G.J., De Guio, F., de Leeuw, F.E., Duchesne, S., Düring, M., Frayne, R., Ikram, M.A., Jouvent, E., MacIntosh, B.J., 2019. Harmonizing brain magnetic resonance imaging methods for vascular contributions to neurodegeneration. Alzheimer's Dement. Diagnosis. Assess. Dis. Monit. 11, 191-204.

Smith, S.M., 2002. Fast robust automated brain extraction. Hum. Brain Mapp. 17, 143-155.

Soriano-Raya, J.J., Miralbell, J., López-Cancio, E., Bargalló, N., Arenillas, J.F., Barrios, M., Cáceres, C., Toran, P., Alzamora, M., Dávalos, A., Mataró, M., 2014. Tract-specific fractional anisotropy predicts cognitive outcome in a community sample of middle-aged participants with white matter lesions. J. Cereb. Blood Flow Metab. 34, 861-869. https://doi.org/10.1038/jcbfm.2014.26.

Spilt, A., Goekoop, R., Westendorp, R.G.J., Blauw, G.J., De Craen, A.J.M., Van Buchem, M.A., 2006. Not all age-related white matter hyperintensities are the same: a magnetization transfer imaging study. Am. J. Neuroradiol. 27, 1964-1968.

Tate, D.F., Jefferson, A.L., Brickman, A.M., Hoth, K.F., Gunstad, J., Bramley, K., Paul, R. H., Poppas, A., Cohen, R.A., 2008. Regional white matter signal abnormalities and cognitive correlates among geriatric patients with treated cardiovascular disease. Brain Imaging Behav. 2, 200-206. https://doi.org/10.1007/s11682-008-9032-5.

Van den Heuvel, D.M.J., Ten Dam, V.H., De Craen, A.J.M., Admiraal-Behloul, F., Van Es, A., Palm, W.M., Spilt, A., Bollen, E., Blauw, G.J., Launer, L., 2006. Measuring longitudinal white matter changes: comparison of a visual rating scale with a volumetric measurement. Am. J. Neuroradiol. 27, 875-878.

van Straaten, E.C.W., Harvey, D., Scheltens, P., Barkhof, F., Petersen, R.C., Thal, L.J., Jack, C.R., DeCarli, C., 2008. Periventricular white matter hyperintensities increase the likelihood of progression from amnestic mild cognitive impairment to dementia. J. Neurol. 255, 1302.

Van Walderveen, M.A.A., Kamphorst, W., Scheltens, P.H., Van Waesberghe, J., Ravid, R., Valk, J., Polman, C.H., Barkhof, F., 1998. Histopathologic correlate of hypointense lesions on T1-weighted spin-echo MRI in multiple sclerosis. Neurology 50, $1282-1288$.

Vavasour, I.M., Meyers, S.M., Mädler, B., Harris, T., Fu, E., Li, D.K.B., Traboulsee, A., MacKay, A.L., Laule, C., 2019. Multicenter measurements of T1 relaxation and diffusion tensor imaging: intra and intersite reproducibility. J. Neuroimaging 29, 42-51.

Wardlaw, J.M., Smith, E.E., Biessels, G.J., Cordonnier, C., Fazekas, F., Frayne, R., Lindley, R.I., T O'Brien, J., Barkhof, F., Benavente, O.R., 2013. Neuroimaging standards for research into small vessel disease and its contribution to ageing and neurodegeneration. Lancet Neurol. 12, 822-838.

Wardlaw, J.M., Valdés Hernández, M.C., Muñoz-Maniega, S., 2015. What are white matter hyperintensities made of? Relevance to vascular cognitive impairment. J. Am. Heart Assoc. 4, e001140.

Zhang, Y., Brady, M., Smith, S., 2001. Segmentation of brain MR images through a hidden Markov random field model and the expectation-maximization algorithm. IEEE Trans. Med. Imaging 20, 45-57.

Zsoldos, E., Mahmood, A., Filippini, N., Suri, S., Heise, V., Griffanti, L., Mackay, C.E., Singh-Manoux, A., Kivimäki, M., Ebmeier, K.P., 2020. Association of mid-life stroke risk with structural brain integrity and memory performance at older ages: A longitudinal cohort study. Brain Commun. 